\title{
On the local homogeneity and the invertibility
} of a topological space

by

Chung-wu Ho (Edwardsville, III.)

Abstract. The author introduces a concept of local homogeneity for topological spaces and uses it to characterize those invertible spaces which are homogeneously invertible. He then establishes the local homogeneity and the homogeneous invertibility of some spaces and finally compares the local homogeneity of this paper with some other types of local homogeneity which appear in the literature.

1. Introduction. In this paper we shall study a type of local homogeneity of a topological space and its relationship to the invertibility of the space. In particular, we shall answer a question recently raised by J. Chew [3, p. 621] that whether a homogeneously invertible Hausdorff space needs be normal (see Section 4). All the basic definitions are given in Section 2, where we shall study the locally homogeneous spaces and use the concept of local homogeneity to characterize those invertible spaces which are homogeneously invertible. In Section 3, we shall establish the local homogeneity and the homogeneous invertibility of many familiar spaces. Finally in Section 4, we shall look at Chew's question and compare our notion of local homogeneity with some other types of local homogeneity which appear in the literature.

2. Local homogeneity and invertibility. Let $X$ be a topological space. A point $x \in X$ is called a local homogeneous point of $X$ if for each open set $U$ containing $x$, there exists an open set $V$ with the properties that $x \in V \subset U$ and for each $y \in V$, there exists a homeomorphism $f$ of $X$ onto $X$ carrying $x$ into $y$ such that $f(V) \subset U$. Note that we do not require $f$ to be identity outside $U$. A space is said to be locally homogeneous if every point of the space is a local homogeneous point. Observe that local homogeneous points are preserved by homeomorphisms of the space. For any space $X$, the set of all the local homogeneous points is open in $X$. If $X$ is near homogeneous (i.e. if for each nonempty open subset $U$ of $X$ and each $x \in X$, there exists a homeomorphism $f$ of $X$ onto $X$ such that $f(x) \in U$ [2], [10]) and if $X$ has at least one local homogeneous point then the set of all the local homogeneous points of $X$ is open and dense in $X$. There are spaces which have no local homogeneous points. This is clear because there are nontrivial spaces for which the only 
homeomorphism of the space onto itself is the identity homeomorphism [9]. In the following, we shall describe, however, a near homogeneous space which does not contain any local homogeneous point.

Start with a closed interval of unit length: At the midpoint of this interval erect an interval of length $\frac{2}{3}$ so that the two intervals are perpendicular bisectors of each other. At the midpoint of each of the four intervals in the resulting figure, erect an
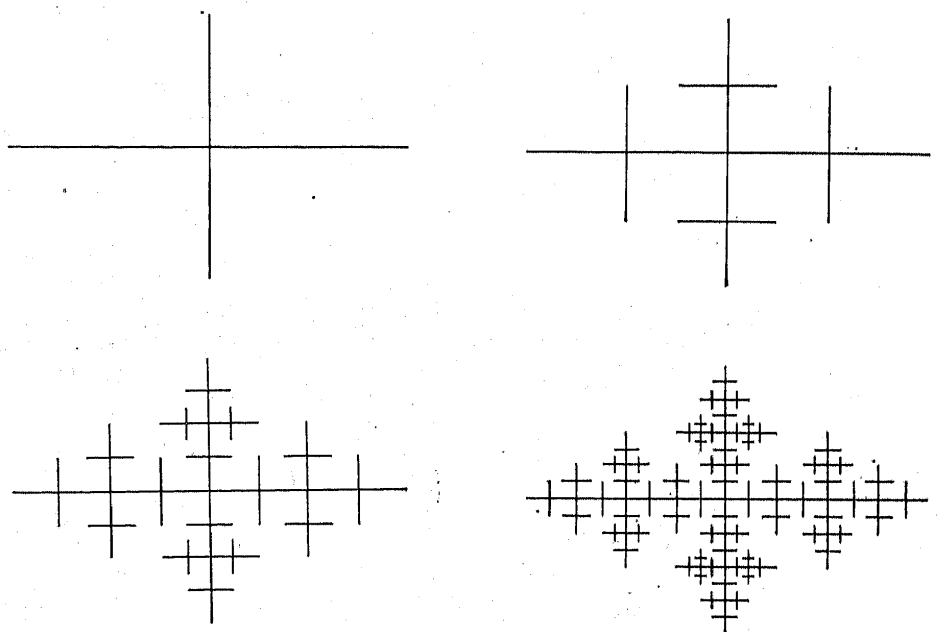

interval whose length is $\frac{2}{3}$ that of the interval being bisected. Continuing this process and taking the closure of the union of those increasing continua, we get a space which is a plane continuum. This space was constructed by P. H. Doyle and J. G. Hocking [5, p. 962-963] in their study of invertible spaces. From their work, we know that this space is near homogeneous. On the other hand, it cannot contain any local homogeneous point, for it has a dense set of cut points and a dense set of noncut points. In any nonempty neighbourhood, a cut point cannot be carried into a noncut point by a homeomorphism.

It is easy to see that a connected locally homogeneous space is always homogeneous in the sense that for any pair of points $x$ and $y$ of the space, there is a homeomorphism of the space onto itself which carries $x$ into $y$. Hence, each locally homogeneous space consists of homogeneous components.

QUESTION 2.1. When is a homogeneous space locally homogeneous?

Our interest in the local homogeneous space stems from the fact that the property of being locally homogeneous characterizes those invertible spaces which are homogeneously invertible (see Theorem 1.5 below). Recall that a space $X$ is said to be invertible at a point $x \in X$ if for each open neighbourhood $U$ of $x$, there exist a homeomorphism $f$ of $X$ onto $X$ such that $f(X-U) \subset U$, and $X$ is said to be homogeneously invertible at a point $x \in X$ if for each open neighbourhood $U$ of $x$ and for each point $y \in X-U$, there exists a homeomorphism $f$ of $X$ onto $X$ such that $f(X-U) \subset U$ and $f(y)=x$. A space is called an invertible space (or homogeneously invertible space) if it is invertible (or homogeneously invertible) at each of its points.

Popos 2. If a space is invertible at a local homogeneous point, then the space is locally homogeneous and homogeneously invertible everywhere.

Proof $X$ be a space which is invertible at a local homogeneous point $x \in X$. Consider an arbitrary point $y \in X$. We contend that $y$ can be carried into $x$ by a homeomorphism of $X$. First choose a neighbourhood $U$ of $x$ such that each point of $U$ can be carried into $x$ by a homeomorphism of $X$. If $y$ belongs to $U$ then our contention is true. If $y$ does not belong to $U$, then by the invertibility of $X$ at $x$, we may choose a homeomorphism $f$ of $X$ which carries $y$ into $U$. Then choose a homeomorphism $g$ of $X$ which carries $f(y)$ into $x$. The composite $g \circ f$ is a homeomorphism of $X$ carrying $y$ into $x$. Since each point of $X$ can be carried into $x$ by morphism of $X$ car $X$ is homogeneous. In a similar way, we can also show that $X$ is homogeneously invertible at $x$. But the properties of being a local homogeneous homogencoun under point and of being a homogeneously invertible point are both preserved under

homeomorphisms. He
invertible everywhere.

We can now give a very simple proof to the following fact which is undoubtedly known.

COROLLARY 2.3. The only manifold which is homeomorphic to a susperision is a sphere.

Pro of. It is well known that spheres are suspensions. Let $M$ be a manifold which is homeomorphic to a suspension. Then $M$ has at least two invertible points (see [6, Theorem 8]). But a manifold is always locally homogeneous. By Proposition 2.2, $M$ is invertible and hence, a topological sphere [4].

PROposirion 2.4. If $X$ is a homogeneously invertible space with at least two disjoint nonempty open subsets, then $X$ is also locally homogeneous.

Proof. We will use the fact that the property of being a local homogeneous point is preserved by homeomorphisms. Since a homogeneously invertible space is point is preser the $X$ is locally homogeneous, it suffices to show clearly homogeneous, to show that $X$ is locally homogeneous, it suffices to show that $X$ contains at least one local homogeneous point. Now, let $W, W^{\prime}$ be two disjoint nonempty open subsets of $X$ with $x \in W$ and $x^{\prime} \in W^{\prime}$. We shall show that $x$ is a local nonempty open subsets of $X$ be any given open set containing $\dot{x}$. Set $V=U \cap W$. homogeneous point. Let $U$ be any 'given open set containing $x$. Set $V$ into $x^{\prime}$ and Then for each $y \in V$ we choose a homogeneously invertible $f$ carrying $y$ into $x^{\prime}$ and $X-W^{\prime}$ into $W^{\prime}$. Also, choose a homogeneously invertible $g$ carrying $x^{\prime}$ into $x$ and $X-V$ into $V$. Then $g \circ f$ is clearly a homeomorphism of $X$ onto $X$ such that $g \circ f(y)=x$ and $g \circ f(V) \subset U$. 
THEOREM 2.5. For a Hausdorff space, local homogeneity is a necessary and sufficient condition for an invertible space to be homogeneously invertible.

Proof. The sufficiency part follows directly from Proposition 2.2. For the necessity part, consider a homogeneously invertible Hausdorff space $X$. If Card $X \leqslant 1$, the space is trivially locally homogeneous. If $\operatorname{Card} X>1$, the space must contain at least two disjoint nonempty open sets. Proposition 2.4 is then applicable.

3. Homogeneously invertible spaces. In this section we shall give some examples of homogeneously invertible spaces. We shall observe that many of the invertible spaces established by Doyle, Hocking et al. in [5] and [6] are in fact homogeneously invertible spaces. In particular, the following are all homogeneously invertible spaces : the $n$-sphere, rational points of the $n$-sphere, an infinite set with the finite complemen topology, the space $R^{\omega}$ of the product of a countable, infinite family of real lines, and the subspace of $R^{\omega}$ consisting of all the infinite sequences of real numbers with finitely many nonzero terms. To show these to be homogeneously invertible spaces, one needs only establish the local homogeneity at a single point. It is straight forward to do this for each of the above spaces. In the following, we shall give a few more examples of homogeneously invertible spaces for which the proof is less straight forward.

Propositron 3.1. The Cantor set is locally homogeneous, and hence, homo: geneously invertible.

Proof. Let $C$ be the Cantor set. We first show the local homogeneity of $C$. At the $n$th stage in constructing the Cantor set from the unit interval, we remove the open middle one-third from each of the subintervals and obtain $2^{n}$ closed subintervals $I_{i}\left(i=1,2, \ldots, 2^{n}\right)$. We shall call each of the sets $I_{i} \cap C\left(i=1,2, \ldots, 2^{n}\right)$ a unit of the Cantor set $C$. Note that each unit is an open and closed subset of $C$ which contains infinitely many further units. Also, each unit of $C$ is homeomorphic to the entire Cantor set $C$ itself.

Now, consider any $x \in C$ and any open set $U$ of $C$ containing $x$. Let $V$ be any unit of $C$ such that $x \in V \subset U$. Since Cantor set is homogeneous (see for instance [11, Ex. 2-37]), for each $y \in V$, there is a homeomorphism $f$ from $V$ onto $V$ which carries $y$ to $x$. Extending $f$ into a homeomorphism $f^{\prime}$ of $C$ by requiring $f^{\prime}$ to be pointwise fixed outside $V$, we get a desired homeomorphism.

Since the Cantor set is known to be invertible [5, p. 959], it is therefore, homogeneously invertible by Proposition 2.2

Proposition 3.2. An infinite product of closed unit intervals is locally homogeneous and homogeneously invertible.

Proof. We first show that an infinite product $X=\prod_{\alpha \in A} I_{\alpha}$ of closed intervals $I_{\alpha}=[0,1]$ is homogeneous. The case when the index set $A$ is countably infinite has long been established [12] (also see [8]). Observe that the case of an uncountable set $A$ follows from the countable case as follows: the space $X$ is homeomorphic to an uncountable product $\prod X_{\beta}$ where each $X_{\beta}$ is a product of a countable, infinite family of closed unit intervals. By the countable case, each $X_{\beta}$ is homogeneous. But a product of homogeneous spaces is obviously homogeneous, hence, $X$ is itself homogeneous.

Now we shall show that the point $x \in X$ with each component $\pi_{\alpha}^{\circ}(x)=\frac{1}{2}$ is a local homogeneous point of $X$ where for each $\alpha \in A, \pi_{\alpha}: X \rightarrow I_{\alpha}$ is the projection map. Let any open set $V$ containing $x$ be given. We can choose a product neighbourhood $U=\bigcap_{i=1}^{n} \pi_{\alpha_{i}}^{-1}\left(U_{i}\right)$ of $x$ in $X$ such that $U \subset V$ and each $U_{i}$ is an open interval in $[0,1]$ containing $\frac{1}{2}$ and having a length less than $\frac{1}{2}$. Now let $y$ be any point in $U$ For each $i=1,2, \ldots, n$, we can clearly choose a (piecewise linear) homeomorphism $h_{i}:[0,1] \rightarrow[0,1]$ which moves the point $\frac{1}{2}$ to the point $\pi_{\alpha_{i}}(y)$ and keeps the set $U_{i}$ in itself. Using the homogeneity of an infinite product space of closed intervals, we may then choose a homeomorphism

$$
k: \prod_{\substack{\alpha \in A \\ \alpha \neq \alpha_{1}, \ldots, \alpha_{n}}} I_{\alpha} \rightarrow \prod_{\substack{\alpha \in A \\ \alpha \neq \alpha_{1}, \ldots, \alpha_{n}}} I_{\alpha}
$$

which carries, for each component $\alpha \neq \alpha_{1}, \alpha_{2}, \ldots, \alpha_{n}$, the point $\frac{1}{2}$ into the point $\pi_{\alpha}(y)$ The cartesian product $h=h_{1} \times h_{2} \times \ldots \times h_{n} \times k$ is then a homeomorphism of $X$ onto itself such that $h(x)=y$ and $h(U) \subset U$. This establishes the local homogeneity of $X$ at $x$. Since $X$ is known to be invertible [5, Theorem 11], it is also homogeneously invertible.

COROLlary 3.3. The Hilbert cube is locally homogeneous and homogeneously invertible.

Propositron 3.4. The Hilbert space is locally homogeneous and homogeneously invertible.

Proof. Since the Hilbert space is known to be invertible [6, Theorem 16], it is sufficient to establish the local homogeneity. But by [1], the Hilbert space is homeomorphic to a product of locally homogeneous spaces, hence, is itself locally homogeneous.

We would like to point out here that not all invertible spaces are homogeneously invertible. For instance, the universal one-dimensional plane curve is known to be invertible [5, pp. 963-964], but it is not homogeneously invertible, for it fails to be homogeneous [13].

4. Separation axioms and other types of local homogeneity. We first consider the relationship between homogeneous invertibility and the separation axioms. James Chew showed that a homogeneously invertible Hausdorff space is always regular [3, Theorem 8$]$. This led naturally to the question that whether such a space needed be normal also. We shall show that it is not necessarily the case. Let $X$ be the product space of an uncountable copies of the open unit interval $(0,1)$. By an argument similar to that used for [6, Theorem 17], one sees easily that $X$ is invertible. It is also easy to see that a product of locally homogeneous spaces is locally homogeneous. Therefore, by Proposition 2.2, $X$ is homogeneously invertible. $X$ is clearly 
Hausdorff (in fact, $X$ is completely regular). The fact that $X$ is not normal follows from a classical theorem of A.H. Stone [15, Theorem 4].

Finally, we shall compare our notion of local homegeneity with some other types of local homogeneity which appear in the literature. One of these, which is very similar to ours, is called strong local homogeneity. It was studied by L. R. Ford [7] and P. S. Mostert [14]. A space $X$ is said to be strong locally homogeneous if for each neighbourhood $U$ of an arbitrary point $x$, there exists a subneighbourhood $V$ such that for each $y \in V$, there exists a homeomorphism $f$ with $f(x)=y$ and with $f$ equal to the identity on $X-U$. A weaker form, introduced by G. S. Ungar [16], which does not insist on $f$ being identity outside $U$ but instead, requires only $f$ to be an " $\varepsilon$-homeomorphism". of the space, is called uniform local homogeneity. Specifically, a space $(X, \mathscr{T})$ is uniformly locally homogeneous if there is a uniformity $\mathscr{A}$ for $X$ such that (1) the uniform topology is $\mathscr{T}$ and (2) given any $x$ in $X$ and any $A \in \mathscr{A}$ there exists a neighbourhood $U$ of $x$ such that if $y \in U$, then there exists a homeomorphism $f$ such that $f(x)=y$ and the graph of $f$ is contained in $A$.

$$
\text { Clearly, strong local homogeneity } \Rightarrow \text { uniform local homogeneity }
$$$$
\Rightarrow \text { local homogeneity. }
$$

On the other hand, it is well known that strong local homogeneity and uniform local homogeneity are not equivalent in general. For instance, the product space of a circle with the Cantor set is not strong locally homogeneous, but is uniformly locally homogeneous. The relationship between uniform local homogeneity and local homogeneity seems to be more complex. Since uniform local homogeneity is defined as a topological property, to construct a counterexample for the equivalence of these two concepts, one has to construct a locally homogeneous space and show that it is not uniformly locally homogeneous under any compatible uniformity. However, it is easy to see that a connected locally homogeneous space is always homogeneous, and in a private communication G. S. Ungar told me that for a locally compact, separable, metric space, homogeneity always implies uniformly locally homogeneity. Thus, for a connected locally compact separable metric space, local homogeneity, homogeneity, and uniform local homogeneity are all equivalent.

Acknowledgment. The author is indebted to G. S. Ungar for pointing out an error in a preliminary version of this paper, and for commenting on the relationships between local homogeneity and uniform local homogeneity.

\section{References}

[1] R. D. Anderson, Hilbert space is homeomorphic to the countable infinite product of lines, Bull. Amer. Math. Soc. 72 (1966), pp. 515-519.

[2] C. E. Burgess, Certain types of homogeneous continua, Proc. Amer. Math. Soc. 6 (1955), pp. 348-350.

[3] J. Chew, Invertibility of function spaces, Amer. Math. Monthly 82 (1975), pp. 618-621.
[4] P. H. Doyle and J. G. Hocking, A characterization of euclidean n-space, Mich. Math. J. 7 (1960), pp. 199-200.

[5] - Invertible spaces, Amer. Math. Monthly 68 (1961), pp. 959-965.

[6] - - and R. P. Osborne, Local invertibility, Fund. Math. 54 (1964), pp. 15-25.

[7] L. R. Ford, Homeomorphism groups and coset spaces, Trans. Amer. Math. Soc. 77 (1954), pp. $490-497$.

[8] M. K. F ort Jr., Homogeneity of infinite products of manifolds with boundary, Pacific J. Math. 12 (1962), pp. 879-884.

[9] J. de Groot, Groups represented by homeomorphism groups I, Math. Ann. 138 (1959), pp. $80-102$.

[10] M. D. Guay, J. G. Hocking and H. V. Kronk, Local near-homogeneity, Amer. Math. Monthly 70 (1963), pp. 827-833.

[11] J. G. Hocking and G. S. Young, Topology, Reading, Massachusetts 1961.

[12] O.-H. Keller, Die Homoiomorphie der kompakten konvexen Mengen im Hilbertschen Raum, Math. Ann. 105 (1931), pp. 748-758.

[13] S. Mazurkiewicz, Sur les continus homogènes, Fund. Math. 5 (1924), pp. 137-146.

[14] P. S. Mostert, Reasonable topologies for homeomorphism groups, Proc. Amer. Math. Soc. 12 (1961), pp. 598-602.

[15] A. H. Stone, Paracompactness and product spaces, Bull. Amer. Math. Soc. 54 (1948), pp. $977-982$.

[16] G. S. Ungar, Local homogeneity, Duke Math. J. 34 (1967), pp. 693-700.

SOUTHERN ILLINOIS UNIVERSITY AT EDWARDSVILLE

Edwardsville, Illinois 